\title{
Development and study of the thermal and electrical behaviour of TGDDS epoxy nanocomposites for high-performance applications
}

\author{
K. Shree Meenakshi • E. Pradeep Jaya Sudhan
}

Received: 21 February 2011/ Accepted: 13 June 2011 / Published online: 28 June 2011

(C) The Author(s) 2011. This article is published with open access at Springerlink.com

\begin{abstract}
The present work focuses on a comparative study of the thermal and electrical behaviour of $\mathrm{N}, \mathrm{N}^{\prime}$ tetraglycidyl diaminodiphenylsulphone (TGDDS) denoted as ' $\mathrm{B}$ ' to find the suitability for its use in high-performance applications. The synthesis of the tetraglycidyl epoxy was done and they were characterized by FT-IR (Fourier transform infrared spectra) and nuclear magnetic resonance spectra $\left({ }^{1} \mathrm{H}-\mathrm{NMR}\right.$ and $\left.{ }^{13} \mathrm{C}-\mathrm{NMR}\right)$. Nanoclay and POSSamine nanoreinforcements denoted as $\mathrm{N} 1$ and $\mathrm{N} 2$ were incorporated into the synthesized epoxy resins. Curing was done using diaminodiphenylmethane (DDM) and bis(3aminophenyl)phenylphosphine oxide (BAPPO) curing agents denoted as $\mathrm{X}$ and $\mathrm{Y}$, respectively. The thermal behaviour of the tetraglycidyl resins and their corresponding nanocomposites was studied by thermogravimetric analysis (TGA). The electrical behaviour, namely dielectric strength, comparative tracking index (CTI), volume resistivity, surface resistivity and arc resistance of the nanocomposites were also studied and the interesting results obtained are discussed.
\end{abstract}

Keywords Epoxy resin - Thermal stability .

Electrical behaviour . Thermogravimetric analysis

\section{Introduction}

Nanotechnology has created a key revolution in the twentyfirst century exploiting the new properties, phenomena and functionalities exhibited by matters when dealt at the level

K. Shree Meenakshi $(\bowtie) \cdot$ E. Pradeep Jaya Sudhan

Department of Chemistry, Anna University,

Chennai 600025, India

e-mail: shreemeenakshik@gmail.com of few nanometers. At this level, the physical, chemical and biological properties of materials differ in fundamental and valuable ways from properties of individual atoms and molecules or bulk matter. Research and development in nanotechnology is directed towards understanding and creating new materials, devices and systems that exploit these new properties. Epoxy resins have several excellent properties such as excellent toughness, adhesion and chemical resistance (Clayton 1988; Kornmann et al. 2002; Potter 1970; May and Tanaka 1973; Bouer 1979; Lee and Neville 1967; Mark and Gaylord 1969; Yee and Pearson 1986; Kinloch et al. 1983). However, they do not possess adequate thermal and mechanical properties to meet the requirements of high-performance structural products. Hence, modification of epoxy resins using suitable modifiers such as phosphorus, sulphone, silicone, polyhedral oligomeric silsesquioxanes (POSS) and nanoclay is mandatory. Demands for epoxy resins are extremely high because of their wide application as adhesives, coatings and as advanced composites in aerospace and electronic industries (Hergenrother et al. 2005, Choi et al. 2001). Incorporation of sulphone unit into the epoxy resin makes the resin become transparent, helps the resin withstand exposure to water, chemicals, increases the resistance of the resin to thermal oxidation and leads to easy processing.

Polyhedral oligomeric silsesquioxanes (POSS) reagents are emerging as a new chemical technology for the nanoreinforced organic-inorganic hybrids and the polymers incorporating POSS monomers are becoming the focus for many studies due to the simplicity in processing and the excellent mechanical properties, thermal stability and flame retardation ( $\mathrm{Li}$ et al. 2001). POSS are a family of nanoscale inorganic cage structures containing a robust silicon/oxygen framework that are intermediate between silica $\left(\mathrm{SiO}_{2}\right)$ and silicone $\left(\mathrm{R}_{2} \mathrm{SiO}\right)$. POSS can be easily 
incorporated into common plastics by means of copolymerization, blending or grafting. Incorporation of POSS into polymers like acrylics, styryls, epoxy and polyethylene has led to enhancements in thermal stability, mechanical properties, glass transition, degradation temperatures, oxygen permeability, reduced flammability and heat evolution as well as modified mechanical properties relative to conventional organic systems (Choi et al. 2001).

Although the organic-clay complexes have been recognized for a long time, the interest in studying these layered silicate materials as nanoscale-reinforcing agent for polymeric materials has only been developed recently. The introduction of as low as 1-5 wt. \% of montmorillonite (MMT) into polymer matrix is well known to result in significant improvement in mechanical strength due to nanometric dimensions and high aspect ratio (Gacitua 2005). The nanoscopic phase distribution as well as synergism between polymer and the layered silicate results in additional properties, such as flame retardation, enhanced barrier properties and ablation resistance. Layered silicates are proven to possess excellent barrier resistance against movement of water or gas molecules and other chemicals due to their high aspect ratio. The present work focuses on a study of the thermal and electrical behaviour of TGDDS nanocomposites to find their suitability for use in highperformance applications.

\section{Experimental}

\section{Materials}

All chemicals were of reagent grade and were used without further purification. 4, 4'-diamino diphenyl methane (DDM) was obtained from Huntsman, USA. Epichlorohydrin and sodium hydroxide were obtained from SD Fine chemicals, India. Triphenylphosphine oxide, 4,4'-diaminodiphenylsulphone (DDS) were obtained from AlfaAaser, Germany. Aminopropyltrimethoxysilane ( $\gamma$-APS), stannous chloride and sulphuric acid were obtained from Merck (Germany). Tetrahydrofuran (THF) and benzene were obtained from Sisco research laboratories, India. Nanoclay was obtained from Nanocor, USA. Hydrochloric acid was obtained from Hi-pure, India.

Synthesis of $\mathrm{N}, \mathrm{N}^{\prime}$-tetraglycidyl

diaminodiphenylsulphone (TGDDS)

Epichlorohydrin $(6.25 \mathrm{~mol})$ was taken in a 1-1 three-necked round bottom flask provided with a mechanical stirrer, nitrogen atmosphere and a water condenser. This was heated to $50^{\circ} \mathrm{C}$ in an oil bath. Then $1.5 \mathrm{~mol}$ of DDS was added and stirred continuously for $4 \mathrm{~h}$ at about $50-55^{\circ} \mathrm{C}$ temperature under nitrogen atmosphere. Chlorohydrin, the intermediate product, was formed and the excess epichlorohydrin used was distilled off under vacuum. Then $2.84 \mathrm{~mol}$ of $40 \% \mathrm{NaOH}$ solution was added drop-wise for $1 \mathrm{~h}$ at $54^{\circ} \mathrm{C}$. The reaction was continued at this temperature for further period of $1 \mathrm{~h}$. The resulting solution was extracted with chloroform. The organic layer was collected and concentrated at a reduced pressure to get the light brown coloured liquid epoxy product (Shih and Ma 1998; Jagdeesh and Shashikiran 2004). The synthesis of TGDDS is shown in Scheme 1.

Synthesis of phosphorus containing diamine

\section{Synthesis of bis(3-aminophenyl)phenylphosphine oxide} (BAPPO)

Triphenylphosphine oxide $(0.1 \mathrm{M})$ was taken in a $500-\mathrm{ml}$ round bottom flask equipped with stirrer, nitrogen atmosphere and $200 \mathrm{ml}$ of $96 \%$ sulphuric acid was added to it. The reactant was dissolved and the reaction system was cooled to $-5^{\circ} \mathrm{C}$ with an ice/salt bath. A solution $(0.4 \mathrm{M})$ of fuming nitric acid in $100 \mathrm{ml}$ of sulphuric acid was added drop wise over a period of $2 \mathrm{~h}$. The reaction system was kept at room temperature for another $8 \mathrm{~h}$. The reaction mixture was hydrolyzed over ice. After the melting of ice, the mixture was extracted with chloroform and washed with aqueous sodium bicarbonate solution until neutral $\mathrm{pH}$. The solvent was removed. The solid product bis(3-nitrophenol)phenylphosphine oxide (BNPPO) was recrystallized from absolute ethanol.

A calculated amount BNPPO was taken in a 1,000-ml round bottom flask with $180 \mathrm{~g}$ of anhydrous powder tin (II)
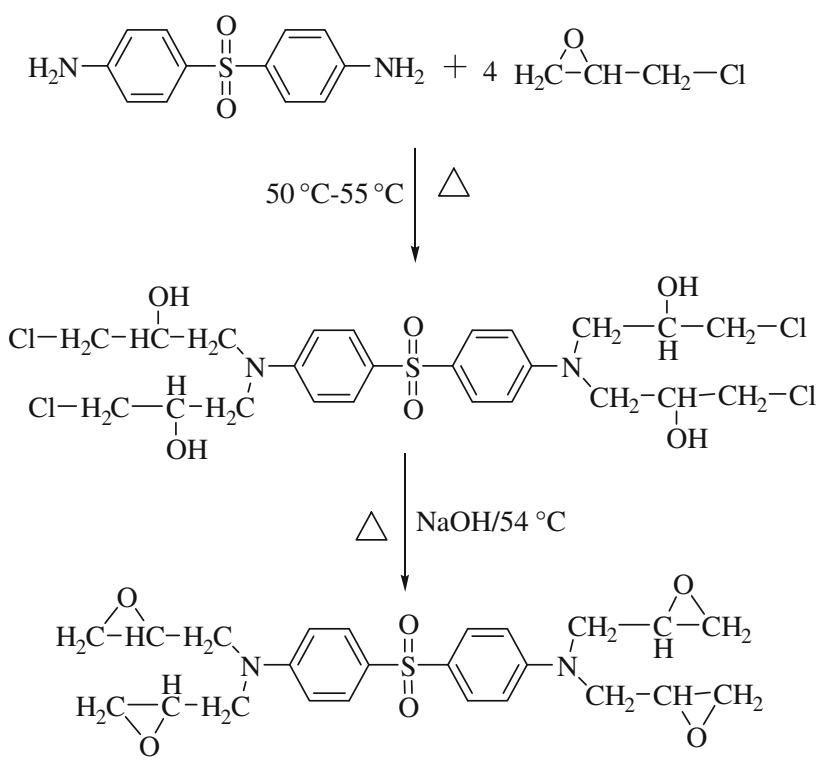

Scheme 1 Synthesis of TGDDS 
chloride. A solution of $200 \mathrm{ml}$ fuming hydrochloric acid in $400 \mathrm{ml}$ ethanol was introduced into the flask. The reaction mixture was stirred at room temperature for $5 \mathrm{~h}$. The solution was concentrated and then neutralized by $25 \%$ of aqueous sodium hydroxide solution. The obtained solution was extracted with chloroform. The organic layer was collected and concentrated under a reduced pressure. The obtained solid was recrystallized from dichloromethane to give a pure product of bis(3-aminophenyl)phenylphosphine oxide (BAPPO). The synthesis of BAPPO (Y) is depicted in Scheme 2.

\section{Synthesis of amine functionalized POSS (POSS-NH 2$)$}

The synthesis of POSS-amine was carried out as follows: stoichiometric amount of POSS-triol $(1.0 \mathrm{~mol})$ dissolved in dry toluene was mixed with aminopropyl triethoxysilane $(1.0 \mathrm{~mol})$ in a $50-\mathrm{ml}$ round bottomed flask and refluxed for $8 \mathrm{~h}$ at $90^{\circ} \mathrm{C}$. The filtrate was then subjected to solvent evaporation to obtain the desired product, which was confirmed by FT-IR and NMR spectra, respectively. The synthesis is shown in the Scheme 3.

\section{Fabrication of resin laminates}

In order to study the properties of the epoxy resins, neat resin laminates were prepared by curing the six different synthesized tetraglycidyl epoxy resins by $\mathrm{X}$ and $\mathrm{Y}$ curing agents as shown in Table 1.

For the fabrication of nanocomposites, the N1 (Nanocor $1.30 \mathrm{E}$ ) was dried at $24 \mathrm{~h}$ at $50^{\circ} \mathrm{C}$ under vacuum. The epoxy resin was mixed mechanically in a reaction vessel with the nanoclay at $50^{\circ} \mathrm{C}$ for $3 \mathrm{~h}$. Then it was further mixed in an ultrasonic bath for $30 \mathrm{~min}$ to disperse the clay in the resin. Later, the mixture was cooled to room temperature in 30 min. The curing agent was then added. After mixing
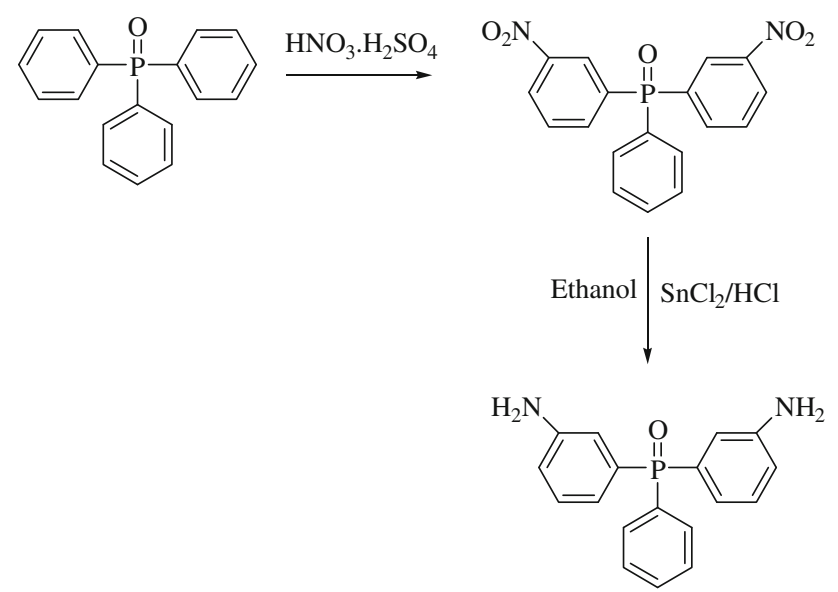

Scheme 2 Synthesis of bis(3-aminophenyl)phenylphosphine oxide (BAPPO) mechanically for $10 \mathrm{~min}$, the mixture was degassed by a vacuum pump to remove the air bubbles and poured into moulds. The nanocomposites were cured for $3 \mathrm{~h}$ at $120^{\circ} \mathrm{C}$ and post-cured for $2 \mathrm{~h}$ at $200^{\circ} \mathrm{C}$. After that the resin plaque was cooled to room temperature naturally. The nanoreinforcement $\mathrm{N} 2$ was mixed with the epoxy resin and then cured. Blends of epoxy, $\mathrm{N} 2$ and curing agent were molten at $100^{\circ} \mathrm{C}$ for $25 \mathrm{~min}$ and then poured into a mould coated with release agent on the inner walls of the mould that was preheated to $120^{\circ} \mathrm{C}$. The curing cycle was $180^{\circ} \mathrm{C}$ for $3 \mathrm{~h}$ and $220^{\circ} \mathrm{C}$ for $2 \mathrm{~h}$. After that the resin plaque was cooled to room temperature naturally, and it was cut into specimens of required dimensions, required for different testing and evaluation studies.

Test methods

The FT-IR spectra were recorded on a Perkin-Elmer 781 infrared spectrometer. ${ }^{1} \mathrm{H}$ NMR spectra was run on a Bruker $400 \mathrm{MHz}$ spectrometer using $\mathrm{CDCl}_{3}$ and DMSO as solvent and tetramethylsilane as internal standard proton decouple. Thermo gravimetric analysis of the polymeric matrices was carried out in TGA-Thermal Analyst Perkin Elmer (TA instrument USA) at a heating of $10^{\circ} \mathrm{C}$ per minute in an inert atmosphere to determine thermal degradation temperature and percentage weight loss. Differential scanning calorimeter NETZSCH DSC 200PC (TA instrument USA) was used to determine the glass transition temperature of the polymer matrices with a heating rate of $10^{\circ} \mathrm{C} / \mathrm{min}$. Dielectric strength was determined as per ASTM D149 in a dielectric strength tester and expressed in $\mathrm{kV} / \mathrm{mm}$. Arc resistance was measured as per ASTM D495 on a test specimen of thickness $3.17 \mathrm{~mm}$. In order to assess the performance of the insulating material, the resistance offered by the polymeric material to electrical current along a $1-\mathrm{cm}^{2}$ surface or through the thickness was determined using a resistivity meter as per ASTM D257. In the comparative tracking index test, the specimen was exposed to either 50 or 100 drops of an aqueous contaminant solution of ammonium chloride and a wetting agent that produced tracking on the surface of the specimen. It was measured as per ASTM D3638.

\section{Results and discussion}

Spectroscopy analysis

\section{FT-IR spectroscopy analysis}

The FT-IR spectrum of TGDDS is shown in Fig. 1. The peaks corresponding to $-\mathrm{S}=\mathrm{O}$ and $-\mathrm{CH}_{2}$ group of TGDDS appear at $1,362,1,184$ and $2,900 \mathrm{~cm}^{-1}$, respectively. 
Scheme 3 Synthesis of amine functionalized POSS (POSS- $\mathrm{NH}_{2}$ )

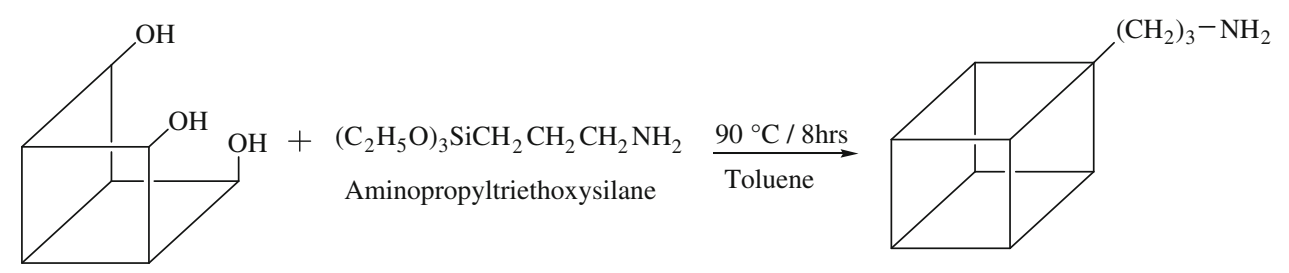

Table 1 Fabrication of neat resin laminates

\begin{tabular}{llll}
\hline Type of epoxy & Matrix name & Nanoreinforcement & Curing agent \\
\hline TGDDS & BX & - & X \\
& BXN1 & N1 & X \\
& BXN2 & N2 & X \\
& BY & - & Y \\
& BYN1 & N1 & Y \\
& BYN2 & N2 & Y \\
\hline
\end{tabular}

Furthermore, absorption peaks which appear at 900-910 $\mathrm{cm}^{-1}$ confirmed the presence of epoxy group in the prepared resin.

The FT-IR spectrum of bis(3-aminophenyl)phenylphosphine oxide (BAPPO) is shown in Fig. 2. The characteristic peaks that appear at 3,499 and $3,393 \mathrm{~cm}^{-1}$ confirm the presence $\mathrm{Ph}-\mathrm{NH}_{2}$ group. The absorption band for $\mathrm{P}-\mathrm{O}-\mathrm{Ph}$ stretching appears at $1,093 \mathrm{~cm}^{-1}$. The peak appearing at $1,277 \mathrm{~cm}^{-1}$ confirms the presence of $\mathrm{P}=\mathrm{O}$ group. Other FT-IR absorption band at $1,498 \mathrm{~cm}^{-1}$ is attributed to the $\mathrm{P}-\mathrm{Ph}$ stretching.

The FT-IR spectrum of POSS- $\mathrm{NH}_{2}$ is depicted in Fig. 3.

The peaks corresponding to $-\mathrm{NH}_{2}$ and $-\mathrm{CH}_{2}$ group of POSS- $\mathrm{NH}_{2}$ appear at 3,500 and 2,900 $\mathrm{cm}^{-1}$, respectively. Furthermore, absorption peaks that appear at 1,200-1,300 and $1,500 \mathrm{~cm}^{-1}$ confirm the presence of $\mathrm{Si}-\mathrm{O}-\mathrm{Si}$ linkage and $-\mathrm{Si}-\mathrm{CH}_{2}$, respectively.

\section{${ }^{1} \mathrm{H}$ and ${ }^{13} \mathrm{C}$ NMR spectrum analysis}

The ${ }^{1} \mathrm{H}$ NMR spectrum of TGDDS is shown in Fig. 4. The signal at $6.5-7.5 \mathrm{ppm}$ maybe due to the aromatic protons.

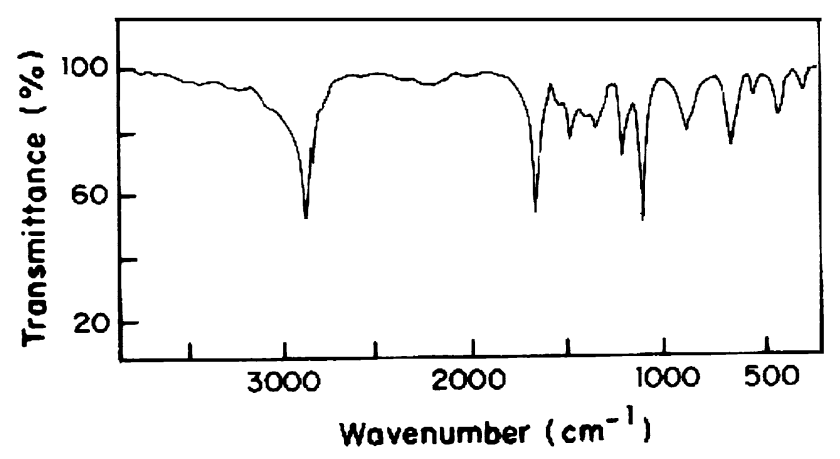

Fig. 1 FT-IR spectra of TGDDS

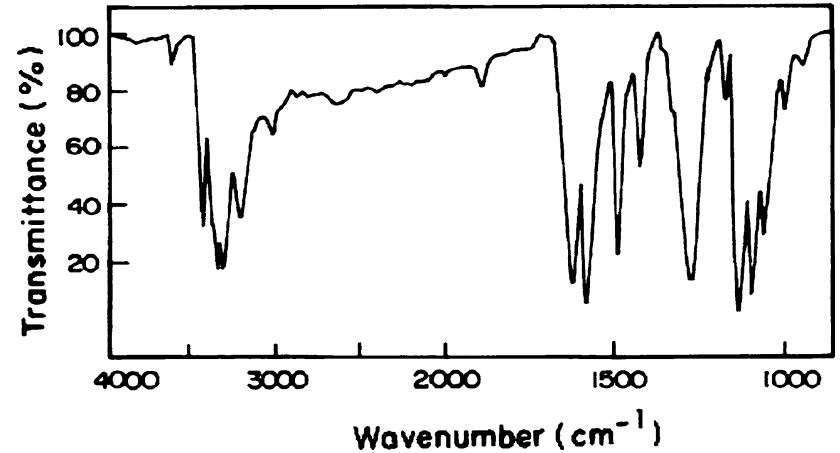

Fig. 2 FT-IR spectra of BAPPO

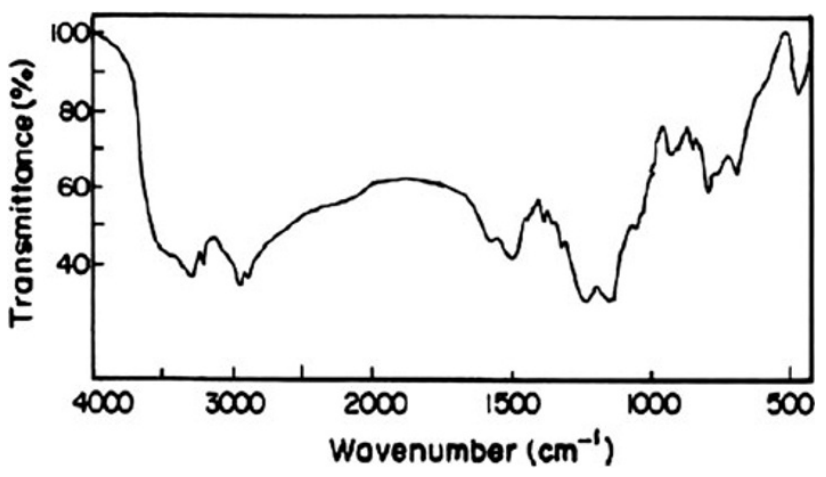

Fig. 3 FT-IR spectra of POSS-amine

The remaining oxirane and methylene protons adjacent to oxirane appear at 3.2-4.5 ppm.

The ${ }^{13} \mathrm{C}$ NMR spectrum of TGDDS is shown in Fig. 5.

The signal at $50 \mathrm{ppm}$ may be due to the presence of - $\mathrm{CH}$ - of epoxy group. The signal which appears at $44 \mathrm{ppm}$ may be due to the $-\mathrm{OCH}_{2}-$ of epoxy group. The signal at about $70 \mathrm{ppm}$ may be due to the presence of $-\mathrm{N}-\mathrm{CH}_{2}-$ carbons. The remaining signal that appears at around 120-140 ppm may be due to aromatic carbons.

The ${ }^{1} \mathrm{H}$ NMR spectrum of BAPPO is shown in Fig. 6. The resonance signal at $3.45 \mathrm{ppm}$ confirms the presence of $-\mathrm{NH}_{2}$ group. The signal at $6.62-7.13 \mathrm{ppm}$ may be due to the aromatic amine proton $\left(\mathrm{C}_{6} \mathrm{H}_{4}-\mathrm{NH}_{2}\right)$. The remaining signal that appears at $7.4-7.9 \mathrm{ppm}$ is due to aromatic protons.

The ${ }^{13} \mathrm{C}$ NMR spectrum of BAPPO is shown in Fig. 7.

The signal at $40 \mathrm{ppm}$ is due to the presence of $-\mathrm{C}-\mathrm{P}$ group. The signal at about $30 \mathrm{ppm}$ may be due to the $-\mathrm{CH}-\mathrm{NH}_{2}-$ of group. The remaining signal appearing at 


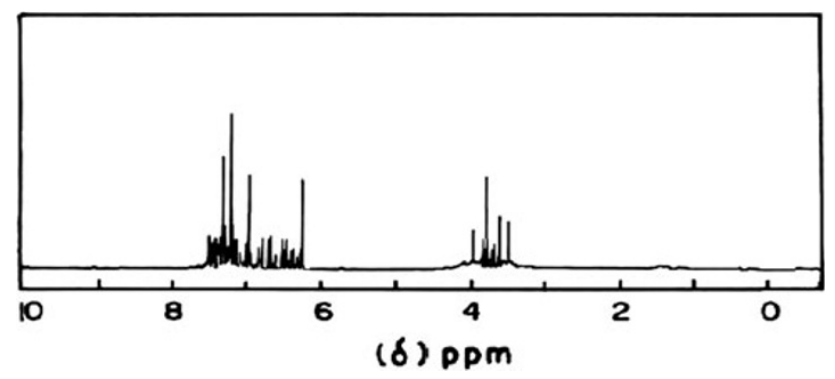

Fig. $4{ }^{1} \mathrm{H}-\mathrm{NMR}$ spectra of TGDDS

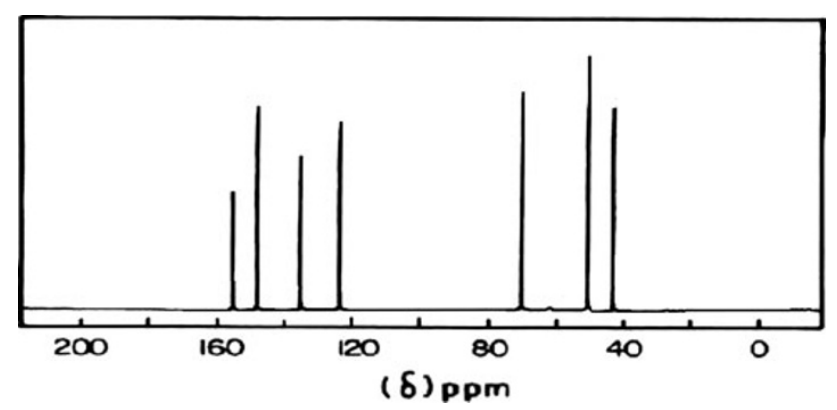

Fig. $5{ }^{13} \mathrm{C}-\mathrm{NMR}$ spectra of TGDDS

around $120-150 \mathrm{ppm}$ is due to aromatic carbons. The single signal in the ${ }^{31} \mathrm{P}$ spectra at around $30 \mathrm{ppm}$ as seen in Fig. 8 confirms the structure of BAPPO.

The ${ }^{1} \mathrm{H}$ NMR spectrum of POSS amine is shown in Fig. 9. The signal at $0.64 \mathrm{ppm}$ is due to the $\mathrm{Si}-\mathrm{CH}_{2}$ protons. The signal at $1.4 \mathrm{ppm}$ corresponds to $\mathrm{C}-\mathrm{NH}_{2}$ protons.

Discussion on thermal stability

\section{Thermogravimetric analysis (TGA)}

The thermal analyses of the resins were studied by thermogravimetric analysis and from the data the thermal degradation temperature of the resins could be found. The char yield and LOI of the resins were found by TGA. The data obtained from the TGA studies is shown in Table 2 and the Figs. 10, 11.

It was observed that the TGDDS systems performed slightly better than the TGDDM systems. The initial

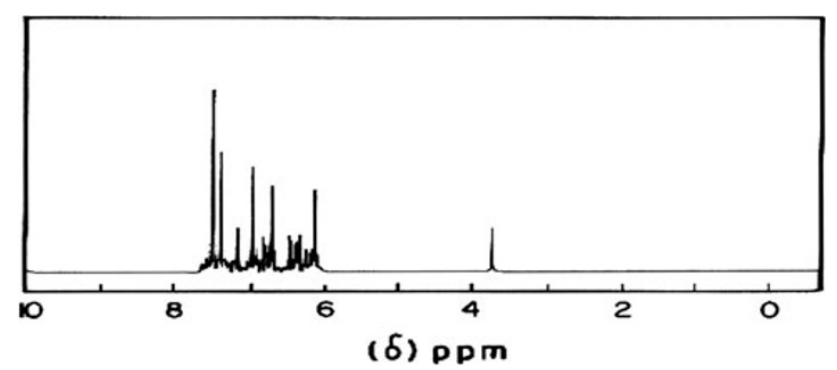

Fig. $6{ }^{1} \mathrm{H}-\mathrm{NMR}$ spectra of BAPPO

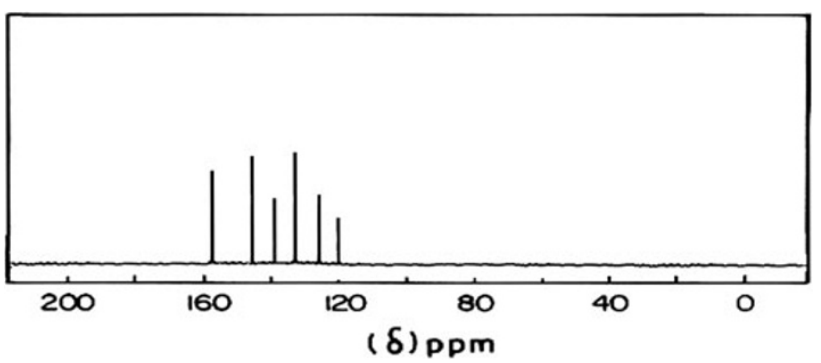

Fig. $7{ }^{13} \mathrm{C}-\mathrm{NMR}$ spectra of BAPPO

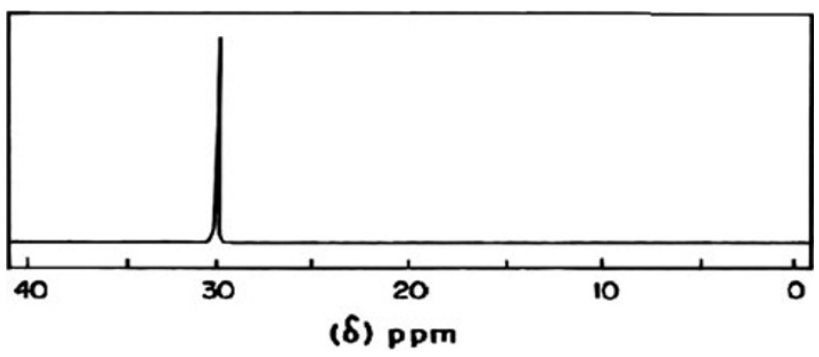

Fig. $8{ }^{31}$ P-NMR spectra of BAPPO

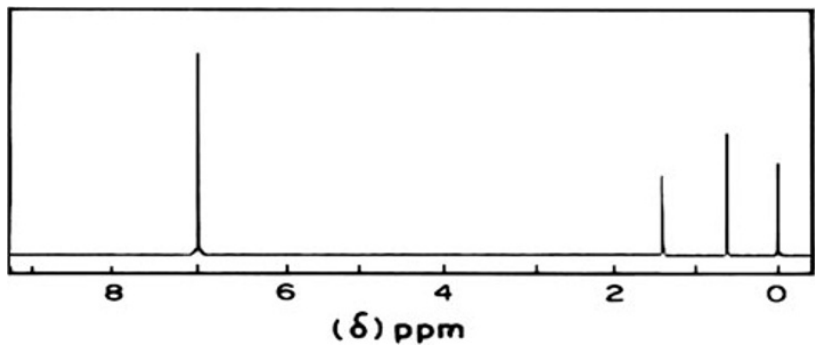

Fig. $9{ }^{1} \mathrm{H}$-NMR spectra of POSS-amine

decomposition temperature, char yield and LOI were higher for the TGDDS systems as seen from the Table 2 .

For example, the initial decomposition temperature of BX system was enhanced to $295^{\circ} \mathrm{C}$ (Fig. 10). The Y cured systems (BY, BYN1 and BYN2) showed a similar double decomposition pattern. In contrast to this observation, the $\mathrm{X}$-cured resin systems (BX, BXN1 and BXN2) showed a single decomposition pattern. The initial degradation temperature of the Y-cured system was lower than that of the $\mathrm{X}$-cured systems, which was due to the decomposition of $\mathrm{P}-\mathrm{O}-\mathrm{C}$ bond at lower temperature. It was interesting to note that the char yield and LOI of the tetraglycidyl systems cured with $\mathrm{Y}$ were found to be higher than those of $\mathrm{X}$-cured systems. For example as seen from Table 2, the char yield of BX was $22 \%$ and LOI was 27, whereas the char yield of BY was $30 \%$ (Fig. 11) and LOI was 31. The higher char yield observed for Y-cured systems may be due to the formation of a protective char layer formed as a result of the degradation that occurred at a lower temperature, thereby protecting the underlying matrix from further degradation. 
Table 2 Data of thermogravimetric analysis (TGA)

\begin{tabular}{llll}
\hline Resin System & $\begin{array}{l}\text { Initial decomposition } \\
\text { temperature }\left({ }^{\circ} \mathrm{C}\right)\end{array}$ & Char yield $(\%)$ & LOI \\
\hline BX & 295 & 22 & 27 \\
BY & 195 & 30 & 31 \\
BXN1 & 310 & 25 & 29 \\
BYN1 & 220 & 33 & 33 \\
BXN2 & 330 & 28 & 30 \\
BYN2 & 235 & 37 & 34 \\
\hline
\end{tabular}

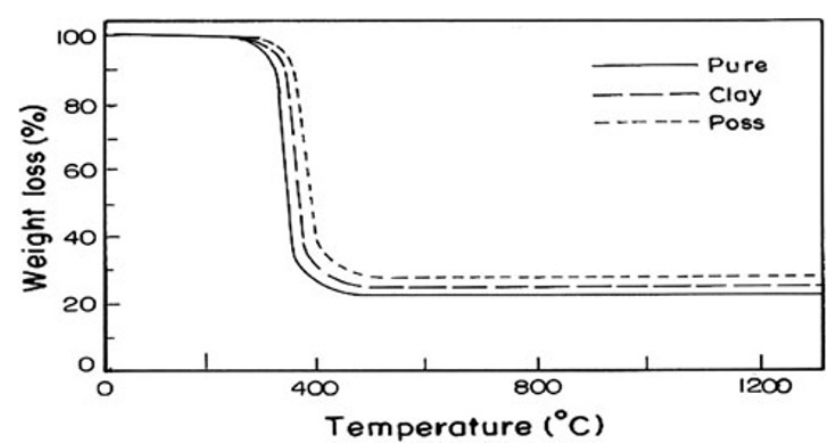

Fig. 10 TGA of BX based systems

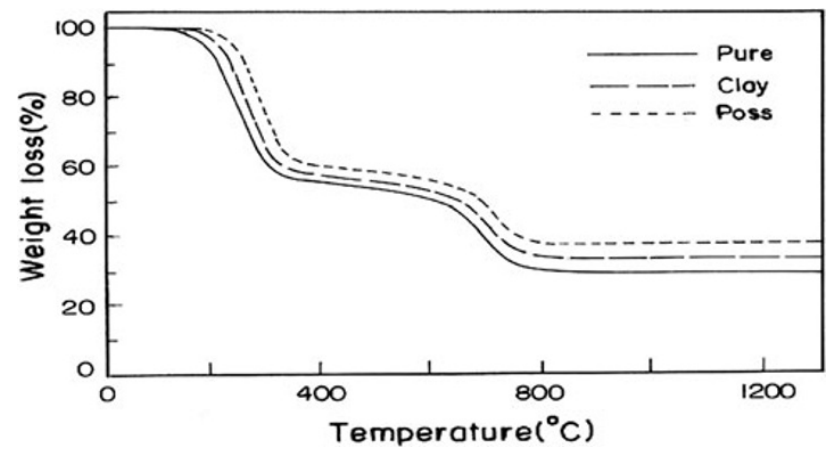

Fig. 11 TGA of BY based systems

The addition of nanoreinforcements produced a significant improvement on the thermal stability, char yield and LOI of all tetra functional epoxy resin systems. For example the initial decomposition temperature of BX system was $295^{\circ} \mathrm{C}$, while the initial decomposition temperatures of BXN1 and BXN2 systems were significantly improved to 310 and $330^{\circ} \mathrm{C}$, respectively, as seen from the Table 2 and Fig. 10. Similarly the char yield for the BX system was $22 \%$ and that for the BXN1 and BXN2 systems was 25 and $28 \%$, respectively, leading to an enhanced LOI values. Incorporation of POSS-amine significantly enhanced the thermal stability of the epoxy resin due to the formation of an inert silica layer on the surface of materials when decomposition takes place and prevented further oxidation of the inner part of the epoxy matrices. POSS molecules having silica like $\mathrm{Si}-\mathrm{O}-\mathrm{Si}$ structure led to higher inorganic components in the cured materials, resulting in higher char yields (Zhang et al. 2007). This Si-O-Si linkage prevented the underlying polymeric matrix from further degradation. On the other hand, the clay nanolayers acted as barriers by preventing the evolution of volatile degradation products from the epoxy matrices (Leszczyńska et al. 2005). Between the two nanoreinforcements, the best results of thermal stability and flame retardancy were given by POSS amine-reinforced system, namely BYN2.

Discussion on electrical properties

The results of the various electrical studies are shown in Table 3.

As seen from the Table 3, the Y-cured systems were found to show better electrical properties than the $X$-cured systems. For example, the dielectric properties of the $\mathrm{X}$-cured system BX was $14.1 \mathrm{kV} / \mathrm{mm}$ while that of the Y-cured system BY was $15.1 \mathrm{kV} / \mathrm{mm}$. This could be due to the presence of rigid aromatic groups of $\mathrm{Y}$ which might have enhanced the hydrophobic behaviour and may also be due to the formation of insulative char layer that contributed to better electrical properties. The incorporation of nanoreinforcements (nanoclay and POSS-amine) was found to enhance the electrical properties significantly. The nanoreinforced epoxy composites yielded breakdown strength values as high as that of the base resin indicating their insulative behaviour required for electrical application. For example surface resistivity of BX system was $3.02 \times 10^{12} \mathrm{Ohm}$, whereas for the system BXN1 and BXN2 the surface resistivity was enhanced to $5.16 \times 10^{12}$ and $7.24 \times 10^{12} \mathrm{Ohm}$, respectively. Similar observation was noticed for the dielectric strength and arc resistance measurements of nanocomposites. This could possibly be due to the presence of the siloxane linkages present in the nanoreinforcements that may be responsible for the enhanced insulative properties.

\section{Conclusion}

The present work deals with the development and characterization of high-functionality epoxy resin, namely TGDDS. These epoxies were cured with DDM and BAP$\mathrm{PO}$, respectively, with and without the incorporation of nanoclay (Nanomer 1.30 E) and nanoreinforcement (POSS amine) to get matrix materials to be utilized for highperformance applications. The developed materials were characterized by spectral studies to ascertain their structures. The polymerization of these resins was carried out in 
Table 3 Electrical properties of various systems

\begin{tabular}{llllll}
\hline Systems & $\begin{array}{l}\text { Dielectric } \\
\text { strength } \\
(\mathrm{kV} / \mathrm{mm})\end{array}$ & $\begin{array}{l}\text { Surface } \\
\text { resistivity } \\
(\mathrm{Ohm})\end{array}$ & $\begin{array}{l}\text { Volume } \\
\text { resistivity } \\
(\mathrm{Ohm})\end{array}$ & $\begin{array}{l}\text { Arc } \\
\text { resistance } \\
(\mathrm{s})\end{array}$ & $\begin{array}{l}\text { Comparative } \\
\text { tracking } \\
\text { index (Volts) }\end{array}$ \\
\hline BX & 14.1 & $3.02 \times 10^{12}$ & $>10^{14}$ & 80 & $>600$ \\
BY & 15.1 & $4.22 \times 10^{12}$ & $>10^{14}$ & 88 & $>600$ \\
BX N1 & 16.3 & $5.16 \times 10^{12}$ & $>10^{14}$ & 89 & $>600$ \\
BY N1 & 17.4 & $6.35 \times 10^{12}$ & $>10^{14}$ & 100 & $>600$ \\
BX N2 & 18.2 & $7.24 \times 10^{12}$ & $>10^{14}$ & 99 & $>600$ \\
BY N2 & 19.4 & $8.18 \times 10^{12}$ & $>10^{14}$ & 108 & $>600$ \\
\hline
\end{tabular}

the presence and absence of nanoclay and nanoreinforcement to get nanocomposites with improved properties ideally suited for advanced engineering applications.

The thermal studies of these materials were carried out by means of TGA and the results obtained from this study clearly indicate that BAPPO (Y) cured tetra epoxies, irrespective of nanoclay and nanoreinforcement showed better char yield and LOI values in spite of their degradation at a lower temperature. The incorporation of nanoclay and nanoreinforcement to both the epoxy improved the IDT, char yield and LOI values. The Y-cured systems were found to show better electrical properties than that of X-cured systems. This could be due to the presence of rigid aromatic groups which enhanced the hydrophobic behaviour and also due to the formation of insulative char layer that contributed to improved electrical properties. The addition of nanoreinforcements (nanoclay and POSS-amine) was found to significantly enhance the electrical properties. For example the arc resistance value of the neat system BX was found to be $80 \mathrm{~s}$, while for the nanocomposites BXN1 and BXN2 the values were enhanced to 89 and $99 \mathrm{~s}$, respectively. This could be attributed to the presence of the siloxane linkages present in the nanoreinforcements that enhanced the insulative properties.

From the data obtained from different studies, it can be concluded that the novel organic-inorganic nano hybrid composites synthesized in the present study, having improved mechanical, thermal properties being self extinguishable, heat resistant at the same time with excellent dielectric properties can very well be used for automotive, electronics and advanced aerospace applications for improved performance and longevity than the materials that are currently in use.

Acknowledgments The authors are grateful to Council for Scientific and Industrial Research (CSIR) and Department of Science and Technology (DST) for having provided financial assistance to carry out this work and thankful to Anna University, Chennai, India, for providing necessary facilities for this work.

Open Access This article is distributed under the terms of the Creative Commons Attribution License which permits any use, distribution and reproduction in any medium, provided the original author(s) and source are credited.

\section{References}

Bouer RS (1979) Epoxy resin chemistry. Advances in Chemistry Series. American Chemical Society, Washington

Choi J, Harcup J, Yee AF, Zhu Q, Laine RM (2001) Organic/ inorganic hybrid composites from cubic silsesquioxanes. J Am Chem Soc 123:11420-11430

Clayton A (1988) Epoxy resins: chemistry and technology. Marcel Decker, New York

Gacitua W (2005) Polymer nanocomposites; synthetic and natural fillers a review. Maderas Cienc Tecnol 7:159-178

Hergenrother PM, Thompson CM et al (2005) Flame retardant aircraft epoxy resins containing phosphorus. Polymer 46:5012-5024

Jagdeesh KS, Shashikiran K (2004) New tetrafuntional epoxy resin system with increased shelf life. J Appl Polym Sci 93:2790-2801

Kinloch AJ, Shaw SJ, Hunston DL (1983) Microstructure and fracture studies. Polymer 32:1341-1354

Kornmann X, Thomann R, Mulhaupt R, Finter J, Berglund LA (2002) High performance epoxy-layered silicate nanocomposites. Polym Eng Sci 42:1815-1826

Lee H, Neville K (1967) Handbook of epoxy resins. McGraw Hill, New York

Leszczyńska A, Njuguna J, Pielichowski K, Banerjee JR (2005) Polymer/montmorillonite nanocomposites with improved thermal properties: part I. Factors influencing thermal stability and mechanisms of thermal stability improvement. Thermochimica Acta 453:75-96

Li G, Wang L, Ni H, Pittman CU (2001) Polyhedral oligomeric silsesquioxane (POSS) polymers and copolymers: a review. J Inorg Organomet Polym 11:123-154

Mark HF, Gaylord NG (1969) Encyclopedia of polymer science and technology. Inter Science, New York

May CA, Tanaka GY (1973) Epoxy resin chemistry and technology. Marcel Dekker, New York

Potter WG (1970) Epoxide resins. Springer, New York

Shih WC, Ma CCM (1998) Tetrafunctional aliphatic epoxy(I). Synthesis and characterization. J Appl Polym Sci 69:51-58

Yee AF, Pearson RA (1986) Toughening mechanism in elastomermodified epoxy resins-mechanical studies. J Mater Sci 21: $2462-2474$

Zhang Z, Liang G, Wang X (2007) The effect of POSS on the thermal properties of epoxy. Poly Bull 58:1013-1020 